\title{
A Review of Therapy for Primary Membranous Nephropathy Post-MENTOR
}

\author{
Vinay Srinivasa
}

\begin{abstract}
Primary membranous nephropathy (PMN) is the most common cause of nephrotic syndrome in adults worldwide. Recent evidence confirms an autoimmune pathogenesis highlighted by the discovery of the phospholipase A2 receptor (PLA2R) antigen. PLA2R serves as both a marker and a guide for treatment. Traditional treatments, including alkylating agents and calcineurin inhibitors, have been associated with adverse risk profiles. Consequently, alternative therapies have been developed. Rituximab is a promising addition to the therapeutic armamentarium of PMN. However, there are few head-to-head trials comparing rituximab with cyclophosphamide. In this clinical review, the results of the MENTOR, STARMEN, and RI-CYCLO trials are discussed and evaluated. Based on the results of these trials, a more personalized treatment approach is needed.
\end{abstract}

Keywords: Primary membranous nephropathy; Personalizing therapy; PLA2R; Rituximab; MENTOR

\section{Introduction}

Primary membranous nephropathy (PMN) is the most common cause of nephrotic syndrome in adults, accounting for $20-40 \%$ of all cases. It is an autoimmune disease with an incidence rate of 1.2 per 100,000 persons per year, and a male to female ratio of $2: 1$ [1-17]. The discovery of autoantibodies to phospholipase A2 receptor (PLA2R) by Beck and Salant in 2009 revolutionized our understanding of the pathophysiology of this unique disease [2].

PLA2R, a normal molecule in the podocyte structure, serves as the target antigen for PLA2R antibodies, causing an immunological response that results in the clinical signs of proteinuria and hypoalbuminemia [1-17]. Furthermore, the identification of this antigen and antibody has had a significant impact on the treatment approach. A serological approach involving evaluation of the PLA2R antibody levels has been

Manuscript submitted July 13, 2021, accepted September 20, 2021

Published online September 28, 2021

General Medicine and Nephrology, Gold Coast Private Hospital, Australia. Email: docvgs@yahoo.com.au

doi: https://doi.org/10.14740/wjnu426 proposed, which serve not only as a marker of disease evolution, but also as a determining factor for treatment $[11,12]$.

Since the discovery of PLA2R, additional autoantibodies have been identified that target glomerular proteins: thrombospondin type 1 domain-containing 7A (THSD7A), exostosin (EXT1/EXT2), neuroepidermal growth-factor-like protein 1 (NELL1), and semiphorin B. The discovery of these autoantibodies has led to a new era of disease diagnosis and treatment, and it is likely that more will be discovered in the next few years [9].

In this clinical review, the results of the MENTOR $[2,9]$, STARMEN [8], and recently published RI-CYCLO [10] trials are discussed and evaluated. The purpose of this review is to illustrate the changing landscape of PMN therapy post-MENTOR, highlighting alternative therapies including rituximab and other agents that have been used more recently instead of the tried and tested cyclical regimen consisting of corticosteroids, alkylating agents, and calcineurin inhibitors. Alkylating agents and calcineurin inhibitors have significant adverse effects, including gonadal toxicity, bone marrow suppression, oncogenic effects, and nephrotoxicity [1-16].

The results from these landmark trials call for a more personalized approach to treatment, moving PMN therapy further into the realms of precision medicine.

Since Ponticelli's seminal paper was published in the New England Journal of Medicine in 1984, cyclical therapy with alkylating agents has been regarded as the gold standard therapy for PMN. This therapeutic approach is effective as remission rates reportedly vary from $77 \%$ to $93 \%$ [6]. Of concern is that these agents are associated with an adverse risk profile that includes malignancy, gonadal toxicity, infection, dermatological manifestations, and bone marrow suppression, thus requiring caution in their use [1-16].

The GEMRITUX study in 2017 was the first randomized controlled trial (RCT) to use rituximab as a treatment for patients with biopsy-proven PMN and nephrotic syndrome. After 6 months of non-immunosuppressive antiproteinuric treatment (NIAT), 37 patients were assigned to receive $375 \mathrm{mg} / \mathrm{m}^{2}$ intravenous rituximab on days 1 and 8 . Overall, 38 patients continued to receive NIAT. Rituximab demonstrated positive effects in terms of its ability to induce proteinuria remission in response to the decline in PLA2R antibodies, suggesting that PLA2R antibodies can be used as a marker of the treatment response [8, 9].

The results from the GEMRITUX study heralded future trials: MENTOR, STARMEN, and RI-CYCLO. These studies provide further support for rituximab as an alternative agent for the treatment of PMN, thus expanding the therapeutic ar- 
mamentarium. Importantly, rituximab has now superseded calcineurin inhibitors in the treatment hierarchy. However, there are important points to be considered when interpreting the results of these trials.

\section{MENTOR}

The MENTOR study was an RCT comparing rituximab and cyclosporine treatments for PMN. Performed in North America, this study included 130 patients with proteinuria (urinary protein excretion of at least $5 \mathrm{~g} /$ day) and creatinine clearance $>40 \mathrm{~mL} / \mathrm{min} / 1.73 \mathrm{~m}^{2}$. Patients were treated with rituximab (two infusions of 1,000 mg each, 14 days apart, repeated at 6 months in the event of a partial response) or cyclosporine (at a dose of $3.5 \mathrm{mg} / \mathrm{kg}$ of body weight per day for 12 months with progressive tapering) $[2,9]$.

The primary outcome of the MENTOR study was a composite of complete or partial remission of proteinuria at 24 months. Rituximab demonstrated non-inferiority at 12 months in $39 / 65(60 \%)$ patients with achievement of remission as opposed to $34 / 65(52 \%)$ patients in the cyclosporine group $[2,9]$. At 24 months, patients receiving rituximab showed superiority with $39 / 65(60 \%)$ patients achieving either complete or partial remission when compared to $13 / 65(20 \%)$ patients in the cyclosporine group $[2,9,12]$. Moreover, $35 \%$ of the patients in the rituximab group were in complete remission, compared to none in the cyclosporine group [2,9]. Furthermore, of the 96 patients $(74 \%)$ who tested positive for PLA2R antibodies, the rate of decline of autoantibodies to PLA2R was faster and of greater magnitude in the rituximab group compared to the cyclosporine group [2].

It should be noted that a stratification of anti-PLA2R levels was not performed; as such, assessing the effectiveness of therapy based on anti-PLA2R levels is difficult [11]. Furthermore, the subjects were removed from the study early and labeled as treatment failures if proteinuria was reduced by $<$ $25 \%$ at 6 months. The authors acknowledge that this action may have been premature, as patients may have developed an immunological response that had not yet manifested as a proteinuric response $[2,11]$. The influence of spontaneous remission on the results is also not known, as there was no placebo control group [11].

The MENTOR study showed that rituximab was superior to cyclosporine in the treatment of PMN. However, $40 \%$ of patients in the rituximab group and $80 \%$ of patients in the cyclosporine group failed to achieve remission at 24 months, prompting questions on the trial design and indicating unmet needs in the treatment $[2,11]$.

\section{STARMEN}

The STARMEN trial was an open-label RCT of 86 patients with PMN and persistent nephrotic syndrome that was conducted in Spain. Patients were assigned to receive either a 6-month cyclical therapy consisting of corticosteroids and cyclophosphamide, or sequential treatment with tacrolimus (full dose for 6 months and tapering for 3 months) and rituximab (1 g at month 6) [7].

The underlying hypothesis of the STARMEN trial was to prove that sequential therapy with a calcineurin inhibitor and rituximab was superior to a cyclical regimen of corticosteroids and cyclophosphamide in achieving and maintaining longterm remission in PMN [3]. The inclusion criteria for this study were as follows: patients with at least $>4 \mathrm{~g}$ /day of proteinuria and an estimated glomerular filtration rate (eGFR) of $>45 \mathrm{~mL} /$ $\mathrm{min} / 1.73 \mathrm{~m}^{2}$ [7]. Overall, 43 patients in each group were assigned to receive either 6-month cyclical treatment with a corticosteroid (total cumulative dose of oral methylprednisolone, $3.4 \pm 0.9 \mathrm{~g}$ and intravenous methylprednisolone, $8.2 \pm 1.4 \mathrm{~g}$ ) and cyclophosphamide (total cumulative dose, $10 \pm 3.5 \mathrm{~g}$ ), or sequential treatment with tacrolimus and rituximab $[7,8]$.

The results showed that $77 \%$ of recruited patients were PLA2R positive $[7,8]$. The primary outcome was complete or partial remission of nephrotic syndrome at 24 months. This was achieved in $36 / 43$ patients $(83.7 \%)$ in the cyclical regimen group and in $25 / 43$ patients $(58.1 \%)$ in the tacrolimus-rituximab group [7]. Complete remission at 24 months occurred in $26 / 43$ patients $(60 \%)$ in the cyclical regimen group compared to $11 / 43$ patients $(26 \%)$ in the tacrolimus-rituximab group [7]. Moreover, the number of complete remissions increased after infusion with rituximab [7].

Most notably, the PLA2R antibody titers decreased in both groups. The proportion of anti-PLA2R-positive patients who were able to achieve an immunological response (depletion of anti-PLA2R antibodies) was significantly higher at 3 and 6 months in the corticosteroid-cyclophosphamide group (77\% and $92 \%$, respectively) than in the tacrolimus-rituximab group (45\% and $70 \%$, respectively) [7].

In addition, serious adverse effects were similar in both groups $[7,8]$. The observed superiority of the cyclical regimen over sequential therapy may have been a result of the lower dose of rituximab administered in this trial ( $1 \mathrm{~g}$ as opposed to 2 - $4 \mathrm{~g}$ in MENTOR) [9].

It should also be noted that the majority of the patients who were in the sequential treatment arm had higher baseline anti-PLA2R levels, higher interquartile proteinuria, and lower interquartile albuminuria compared to patients who received the cyclical regimen [7]. Although the study authors deemed this result not significant, they acknowledged that these differences at baseline may have biased the results against the sequential therapy arm [7]. Furthermore, in clinical practice, tacrolimus and rituximab are simultaneously administered, rather than the sequential therapy approach adopted in this trial, casting doubts on the applicability of the latter approach in regular clinical practice.

\section{RI-CYCLO}

The RI-CYCLO trial was a pilot randomized trial performed in Italy, comparing rituximab with a cyclic regimen in patients with PMN and severe proteinuria. In all, 74 patients with PMN and proteinuria ( $>3.5 \mathrm{~g}$ /day) were randomly assigned to receive either rituximab at a dose of $1 \mathrm{~g}$ on days 1 and 15 , or a 
6-month cyclical regimen of corticosteroids, which were alternated with cyclophosphamide after a run-in period of 3 months [10].

The study's inclusion criteria were as follows: proteinuria (urine protein clearance rate of at least $3.5 \mathrm{~g} /$ day) and an eGFR $>30 \mathrm{~mL} / \mathrm{min} / 1.73 \mathrm{~m}^{2}$ [10]. Unlike what was observed in the MENTOR and STARMEN studies, the primary outcome of this study was complete remission of proteinuria at 12 months [10]. Secondary outcomes were the achievement of complete or partial remission at 24 months and the occurrence of adverse effects [10].

Overall, six of 37 patients $(16 \%)$ in the rituximab arm achieved complete remission, compared with $12 / 37$ patients in the cyclical regimen arm [10]. Furthermore, 23 of 37 patients $(62 \%)$ in the rituximab arm, and 27 of 37 patients $(73 \%)$ in the cyclical regimen arm demonstrated complete or partial remission [10].

In brief, there were no benefits or harm reductions associated with rituximab when compared to a cyclical regimen of corticosteroids and cyclophosphamide [10]. Moreover, based on intention-to-treat analysis, the 1-year probability of complete remission of proteinuria was lower in the rituximab group in the per-protocol analysis [10]. Significantly, 20-40\% of the patients failed to respond to rituximab therapy [10].

It should be mentioned that the subjects' baseline PLA2R antibody levels in this trial were lower than those of the subjects in the MENTOR study, although a different assay was used for measurement [10]. Furthermore, the proteinuria cutoff was higher in the MENTOR study [10].

The authors also admitted that the study findings may not be applicable to patients with high levels of anti-PLA2R antibodies, as the duration of the study was too short to capture long-term toxicity [10]. Furthermore, recruitment was slow, and a blinding procedure was not incorporated, which may have introduced bias $[9,10]$.

\section{Unanswered Questions}

Based on the results of the MENTOR study, rituximab was proposed to be superior to cyclosporine for the treatment of PMN $[10,12]$. However, the results from the STARMEN and RI-CYCLO studies showed that it would be difficult to justify a clinical trial that supports rituximab over cyclophosphamide $[9,10]$, especially since unanswered questions still remain. First, the optimum dose of rituximab, and how its response is monitored via measurement of CD 19 levels have areas of uncertainty $[2,7,9,12]$. Second, immunological monitoring of PLA2R-associated membranous nephropathy appears to be a reasonable approach to guide therapy. However, one might wonder how PLA2R-negative patients are monitored [4]. Furthermore, one might ask whether redosing should be guided by the clinical response or proteinuria [4].

Similarly, with newer antigens being discovered, it needs to be determined whether these antigens can be used as markers of disease activity. Can anti-PLA2R levels be used for this purpose? Further validation of serological/histological markers is required.
Relapses are also frequently seen in patients with low PLA2R antibody levels [4]. In such cases, one might wonder whether fixed maintenance therapy should be employed [4].

Furthermore, both the MENTOR and RI-CYCLO studies have shown that rituximab may not be suitable for all patients. According to Scolari et al, recognizing this group and identifying markers of poor prognosis remains an ongoing challenge [13].

\section{Future Therapies}

In this section, potential new therapeutic agents are briefly discussed. A detailed discussion of these agents is beyond the scope of this review. No RCTs exist in this area; as such, the evidence is only supported by case series or case reports.

\section{New CD20 agents}

A one-size-fits-all approach does not work for all patients being treated for PMN. As described above, rituximab may not be effective in certain cases.

Ofatumumab is a new monoclonal antibody directed against CD20. It differs from rituximab as it has different target epitopes $[9,12]$. In addition to acting on the same epitope that is recognized by rituximab, it targets an additional epitope located on a portion of the large extracellular loop of CD20 along with an epitope localized on the small loop of CD20 [9, 12]. A case series reported by Podesta et al described achievement of clinical remission with ofatumumab in patients who were resistant to rituximab with PMN [9].

Sethi et al also recently reported the use of obinutuzumab in patients with PMN, who did not respond to rituximab therapy in a single-center case series [14].

Like ofatumumab, the target epitope on CD20 for obinutuzumab is different from that for rituximab. Moreover, obinutuzumab reportedly induces a greater B-cell apoptotic response compared to rituximab $[11,12]$.

\section{Anti-B-cell activating factor (BAFF) therapy (belimum- ab)/anti-plasma cell therapy}

Belimumab is a human immunoglobulin (Ig) G1-lambda monoclonal antibody that inhibits BAFF [9]. This agent has been approved for the treatment of systemic lupus erythematosus. The recent BLISS trial showed that patients with lupus nephritis who received belimumab together with standard therapy had a better renal response than those who received standard therapy alone [9].

Belimumab was also used as an anti-proteinuric agent in an open-label, prospective, single-arm study comprising 14 patients with PLA2R-positive PMN [1]. The findings illustrated that treatment with belimumab caused a reduction in proteinuria and circulating PLA2R antibody titers by $86 \%$ and $97 \%$, respectively [1].

It has been hypothesized that the advanced stages of PMN 
could be driven by autoreactive plasma cells, which lack CD19 and CD20, thereby making them resistant to rituximab $[9,12]$. Since these cells express CD38, they can serve as a target for anti-CD38 antibodies such as daratumumab and isatuximab, which then kill these plasma cells [9, 12].

Bortezomib, a proteasome inhibitor, may deplete plasma cells causing plasma cell apoptosis [12]. Preliminary data suggest that this agent may be useful in the treatment of cases of PMN that are resistant to other therapies [12].

\section{Conclusion}

The disease pathophysiology of PMN has evolved considerably, further enhancing our knowledge and understanding of this distinctive disease. Results from the MENTOR, STARMEN, and RI-CYCLO studies have demonstrated that rituximab is an alternative treatment option that can be used both as a cyclical therapy as well as in conjunction with calcineurin inhibitors for the treatment of PMN.

Patients who are resistant to rituximab therapy or who are at risk of progression to renal failure may require newer agents or cyclical therapy. Risk stratification of patients with PMN using clinical markers such as proteinuria and immunological monitoring of PLA2R antibodies is needed, thereby allowing for a more personalized approach to treatment. Based on review of the current literature, patients with severe proteinuria defined as more than $8 \mathrm{~g} / 24 \mathrm{~h}$, should be considered for cyclophosphamide given its proven benefit. For those patients with proteinuria less than $8 \mathrm{~g}$ in $24 \mathrm{~h}$, rituximab is an alternative option.

\section{Acknowledgments}

None to declare.

\section{Financial Disclosure}

The author declares that they do not have a financial relationship with any commercial entity that has an interest in the subject of this manuscript.

\section{Conflict of Interest}

The author declares that they do not have a conflict of interest.

\section{Author Contributions}

VS devised and drafted the manuscript.

\section{Data Availability}

The data supporting the findings of this study are available from the corresponding author upon reasonable request.

\section{References}

1. Barrett C, Willcocks LC, Jones RB, Tarzi RM, Henderson RB, Cai G, Gisbert SI, et al. Effect of belimumab on proteinuria and anti-phospholipase A2 receptor autoantibody in primary membranous nephropathy. Nephrol Dial Transplant. 2020;35(4):599-606.

2. Beck LH, Jr., Bonegio RG, Lambeau G, Beck DM, Powell DW, Cummins TD, Klein JB, et al. M-type phospholipase A2 receptor as target antigen in idiopathic membranous nephropathy. N Engl J Med. 2009;361(1):11-21.

3. Ferreira F, Nunes AT. New Treatments in membranous glomerulopathy - from the pitfalls of rituximab to a new era of biological treatments. Portuguese Journal of Nephrology \& Hypertension. 2020;34(3):160-164.

4. Fervenza FC, Appel GB, Barbour SJ, Rovin BH, Lafayette RA, Aslam N, Jefferson JA, et al. Rituximab or cyclosporine in the treatment of membranous nephropathy. N Engl J Med. 2019;381(1):36-46.

5. Floege J, Rovin BH. The STARMEN trial: rethinking calcineurin inhibitor therapy in membranous nephropathy. Kidney Int. 2021;99(4):811-813.

6. Gauckler P, Shin JI, Alberici F, Audard V, Bruchfeld A, Busch M, Cheung CK, et al. Rituximab in membranous nephropathy. Kidney Int Rep. 2021;6(4):881-893.

7. van de Logt AE, Wetzels JF. Rituximab is preferable to cyclophosphamide for treatment of membranous nephropathy: CON. Kidney360. 2021.

8. Oliva-Damaso N, Bomback AS. rituximab is preferable to cyclophosphamide for treatment of membranous nephropathy: PRO. Kidney360. 2021.

9. Podesta MA, Ruggiero B, Remuzzi G, Ruggenenti P. Ofatumumab for multirelapsing membranous nephropathy complicated by rituximab-induced serum-sickness. BMJ Case Rep. 2020;13(1):e232896.

10. Rojas-Rivera J, Fernandez-Juarez G, Ortiz A, Hofstra J, Gesualdo L, Tesar V, Wetzels J, et al. A European multicentre and open-label controlled randomized trial to evaluate the efficacy of Sequential treatment with TAcrolimus-Rituximab versus steroids plus cyclophosphamide in patients with primary MEmbranous Nephropathy: the STARMEN study. Clin Kidney J. 2015;8(5):503-510.

11. Ronco P, Plaisier E, Debiec H. Advances in membranous nephropathy. J Clin Med. 2021;10(4):607.

12. Ronco P, Debiec H. Membranous nephropathy: current understanding of various causes in light of new target antigens. Curr Opin Nephrol Hypertens. 2021;30(3):287293.

13. Scolari F, Delbarba E, Santoro D, Gesualdo L, Pani A, Dallera N, Mani LY, et al. Rituximab or cyclophosphamide in the treatment of membranous nephropathy: the RI-CYCLO randomized trial. J Am Soc Nephrol. 2021.

14. Sethi S, Kumar S, Lim K, Jordan SC. Obinutuzumab is effective for the treatment of refractory membranous nephropathy. Kidney Int Rep. 2020;5(9):1515-1518. 
15. Salvadori M, Tsalouchos A. Editor's pick: new aspects of pathogenesis and treatment of membranous glomerulopathy after the MENTOR study. EMJ Nephrol. 2020;8(1):46-53.

16. Trivin-Avillach C, Beck LH, Jr. Management of membra- nous nephropathy after MENTOR. Clin J Am Soc Nephrol. 2020;15(3):415-417.

17. Trujillo $\mathrm{H}$, Alonso M, Praga $M$. New ways of understanding membranous nephropathy. Nephron. 2020;144(6):261-271. 